Chapman University

Chapman University Digital Commons

Pharmacy Faculty Articles and Research

School of Pharmacy

2010

\title{
Development of Cytarabine Prodrugs and Delivery Systems for Leukemia Treatment
}

Bhupender S. Chhikara

University of Rhode Island

Keykavous Parang

Chapman University, parang@chapman.edu

Follow this and additional works at: http://digitalcommons.chapman.edu/pharmacy_articles

Part of the Chemicals and Drugs Commons, Medicinal and Pharmaceutical Chemistry

Commons, and the Pharmaceutics and Drug Design Commons

\section{Recommended Citation}

Chhikara, Bhupender S., and Keykavous Parang. "Development of cytarabine prodrugs and delivery systems for leukemia treatment." Expert opinion on drug delivery 7.12 (2010): 1399-1414. doi: 10.1517/17425247.2010.527330

This Article is brought to you for free and open access by the School of Pharmacy at Chapman University Digital Commons. It has been accepted for inclusion in Pharmacy Faculty Articles and Research by an authorized administrator of Chapman University Digital Commons. For more information, please contact laughtin@chapman.edu. 


\section{Development of Cytarabine Prodrugs and Delivery Systems for Leukemia Treatment}

\section{Comments}

This is a pre-copy-editing, author-produced PDF of an article accepted for publication in Expert Opinion on Drug Delivery, volume 7, issue 12, 2010 following peer review. The definitive publisher-authenticated version is available online at DOI: $10.1517 / 17425247.2010 .527330$.

\section{Copyright}

Informa Healthcare 
Development of cytarabine prodrugs and delivery systems for leukemia treatment 


\section{Abstract}

Importance of the field: Cytarabine is a polar nucleoside drug used for the treatment of myeloid leukemia and non-Hodgkin's lymphoma. The drug has a short plasma half-life, low stability, and limited bioavailability. Overdosing of patients with continuous infusions may lead to side effects. Thus, various prodrug strategies and delivery systems have been extensively explored to enhance the half-life, stability, and delivery of cytarabine. Among the recent cytarabine prodrugs, amino acid conjugate ValCytarabine and fatty acid derivative CP-4055 (in phase 3 trials) have been investigated for the treatment of leukemia and solid tumors, respectively. Alternatively, delivery systems of cytarabine have emerged for the treatment of different cancers. The liposomal-cytarabine formulation (DepoCyt $\left.{ }^{\circledR}\right)$ has been approved for the treatment of lymphomatous meningitis.

Areas covered in this review: Various prodrug strategies evaluated for cytarabine are discussed. Then, the review summarizes the drug delivery systems that have been used for more effective cancer therapy.

What reader will gain: This review provides in-depth discussion of the prodrug strategy and delivery systems of cytarabine derivatives for the treatment of cancer. The design of cytarabine prodrugs and delivery systems provide insights for designing the next generation of more effective anticancer agents with enhanced delivery and stability.

Take home message: Strategies on designing cytarabine prodrug and delivery formulations showed great promise in developing effective anticancer agents with better therapeutic profile. Similar studies with other anticancer nucleosides can be an alternative approach to gaining access to more effective anticancer agents. 
Keywords: cytarabine, prodrug, delivery system, arabinofuranosylcytosine, araC, liposome, nanoparticle, chitosan, fatty acid-cytarabine, amino acid. 


\section{Introduction}

Blood cancers such as leukemia, non-Hodgkin lymphoma, Hodgkin lymphoma, and myeloma are characterized by the abnormal production of malformed lymphocytes and/or other blood cells. The cancer cells multiply without control and accumulate in bone marrow, blood and lymphatic tissues, and thus interfere with healthy cells and immune cell production and function. Ultimately these events result in blood/lymphatic disorders, such as leukemia, lymphoma, myeloma, and myelodysplastic syndromes based on affected tissue [1].

The malformed cells are results of one or more acquired mutations in DNA of lymphatic or blood forming stem cells, which on multiplication (clone formation) generate high number of abnormal white blood cells. For example, in chronic myelogenous leukemia $(\mathrm{CML})$ acquired mutation affects hematopoietic stem cells. $\mathrm{CML}$ is characterized by presence of Philadelphia $(\mathrm{Ph})$ chromosome generated through translocation and fusion of the Abelson oncogene (ABL) at chromosome 9 and the break-point cluster region (BCR) at chromosome 22 leading to the generation of a BCR$\mathrm{ABL}$ fusion oncogene, which in turn translates into a Bcr-Abl oncoprotein [2].

Leukemia, lymphoma, and myeloma are diagnosed in all ages and account for the deaths of approximately 54,000 people in United States in 2010 , nearly 9.5 percent of deaths from cancer in 2010. Leukemia is a one of the most fatal hematopoietic neoplasms and represents a wide range of blood cancers mainly related to abnormal increase in the number of white blood cells (leukocytes). The death toll is expected to increase as new cases are diagnosed annually, suggesting a highly fatal nature of 
leukemia if unattended or untreated at early stages of development [3]. Thus, causes, diagnosis, treatment, and prognosis of leukemia are subjects of major interest. [4].

Depending upon the type of abnormal early blast or mature cells present, leukemia can be categorized into acute or chronic forms, respectively. The acute leukemia is identified by the rapid increase of immature white blood cells that crowd and accumulate around bone marrow, and thus induce the haemopoietic organ inability to produce normal blood cells. Therefore, immediate and aggressive treatment is required in acute leukemia due to rapid progression and accumulation of the malformed cells, which with time, enter the bloodstream and invade other organs. Chronic leukemia is caused by the excessive build up of abnormal mature white blood cells, which takes time to progress [5]. Further, leukemia can be lymphoblastic or lymphocytic leukemias (LL) and myeloid or myelogenous leukemias (ML) depending upon type of blood cell affected. The lymphoblastic or lymphocytic leukemias occur due to the cancerous modifications of bone marrow cells that are converted to lymphocytes. Lymphocytes have roles as the infection-fighting immune system cells. The myeloid or myelogenous leukemias result from the cancerous change of marrow cell that form red blood cells, platelets, and white blood cells $[6,7]$.

Owing to the heterogeneity of leukemia, treatment options for different classes of leukemias vary. The leukemia chemotherapy involves drugs from different classes ranging from kinase inhibitors to DNA synthesis inhibitors [8]. In many cases, a combination of two or more drugs is administered to patients to manage leukemia $[9,10]$. Radiotherapy is sometimes used along with chemotherapy in some types and 
stages of blood cancer, such as acute lymphocytic leukemia (ALL) and B-Cell lymphomas $[11,12]$.

Cytarabine (4-amino-1- $\beta$-D-arabinofuranosyl-2-(1H)-pyrimidinone, 1- $\beta$-Darabinofuranosylcytosine, araC, Cytosar-U) is a pyrimidine nucleoside-based anticancer drug (Figure 1) with arabinose sugar widely used for the treatment of leukemia. Cytarabine is predominantly used against acute myelogenous leukemia (AML) and nonHodgkin's lymphoma (NHL), chronic myelocytic leukemia (blast phase), ALL and erythroleukemia $[13,14]$. It may be used alone or in combination with other anticancer agents, such as daunorubicin, doxorubicin, thioguanine, or vincristine.

When cytarabine is used in combination with other anticancer drugs for the treatment of different leukemias and solid tumors [15,16], the drug combinations generally improve the cancer therapy by synergistic effect [17]. Cytarabine is used in induction therapy in combination with anthracyclines and in consolidation therapy at higher dose for AML patients. The combination of cytarabine with purine nucleoside analogs, such as fludarabine and cladribine, has been extensively explored in the treatment of patients with relapsed or refractory AML [18]. In Hodgkin's disease (HD) and NHL, cytarabine is used in conjunction with other drugs using different regimens, such as DHAP (cytarabine-cisplatin-dexamethasone) [19] and ESHAP (etoposidemethylprednisolone-cytarabine-cisplatin) [20] in patients with relapsed or refractory lymphoma. Cytarabine is used as front line drug for mantle cell lymphoma (MCL) [21]. Hyyper CVAD-MTX/Ara-c regimen (rituximab with hyperfractioned cyclophosphamide, doxorubicin, vincristine and dexamethasone plus methotrexate and cytarabine) is used for Burkitt's leukemia/lymphoma [22] and ALL [23]. 


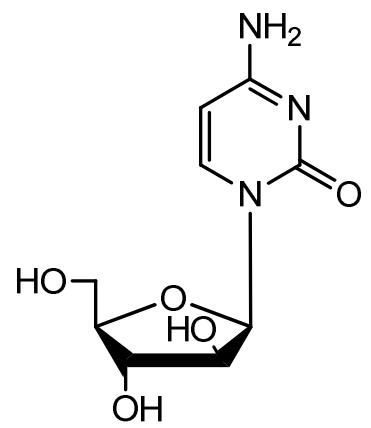

Figure 1. Chemical structure of cytarabine.

Cytarabine acts on rapidly dividing cells and inhibits DNA synthesis at S-phase of cell cycle and also hinders progression of cells from G1 phase to the S-phase. When inside the cell, cytarabine is converted into the triphosphate derivatives by kinase enzymes to show its cytotoxic effect. The exact mechanism of action of cytarabine triphosphate has not been elucidated but it appears to inhibit DNA polymerase by competing with deoxycytidine triphosphate [24] and thus reduces the cell replication.

Cytarabine is a polar nucleoside and has a short plasma half-life. After three phosphorylation step, cytarabine is converted to cytarabine triphosphate. It has been suggested that cytarabine triphosphate inhibits DNA polymerase by competing with the natural substrate, deoxycytidine triphosphate, resulting in DNA synthesis inhibition. The low bioavailability of cytarabine is created by its low permeability across cell membrane and rapid conversion into inactive $1-\beta-D$-arabinofuranosyluracil (AraU). The drug is rapidly converted into its uridine derivative by deamination of cytosine ring in the presence of cytidine deaminase [25]. Thus, continuous intravenous infusion of higher doses is required to maintain constant plasma level of the drug in 8 to $24 \mathrm{~h}$. The higher 
doses of cytarabine lead to toxicity on normal organs and side effects [26]. Alternatively, cytarabine is administered by subcutaneous infusion in which peak plasma levels are attained after 20 to $60 \mathrm{~min}$ and decrease below steady-state level after $100 \mathrm{~min}$ [27].

Understanding the mechanism of the action and metabolism of cytarabine has allowed many investigations to improve the low bioavailability and stability. These efforts, depending upon formulation and modification, can be divided into two major categories: prodrugs and drug delivery systems. The prodrug strategy for cytarabine involves the chemical modification or introduction of a potentiating group on parent drug while the delivery system involved in physical encapsulation of drug without introduction of any chemical modification on parent drug. The molecules synthesized for prodrug evaluation may have higher therapeutic effect while the delivery system may improve the effect in a particular tissue or organ. Various explored prodrug strategies and potential of delivery systems for cytarabine are discussed.

\section{Cytarabine Prodrugs}

Most of the therapeutic drugs have to pass physiological barriers before reaching to the target organ/tissue of action. Many drugs get modified into inactive derivatives during the transportation process. Among the various strategies used to minimize the undesirable metabolism of drug, prodrug approach offers a great promise in retention of pharmacological properties of drugs, improving the drug efficacy, decreasing the toxicity, or reduction in dose of administration.

Prodrugs are defined as pharmacologically inert chemical derivatives that can be converted into the active drug molecules under in vivo physiological conditions through 
an enzymatically or nonenzymatic process, to exert a therapeutic effect at desired target site. An Ideal prodrug is converted to the original therapeutically active form of drug at target organ and the substituted group is eliminated from the body [28]. Thus, prodrug approach may minimize undesirable toxicity related to the parent drug in non-targeted organs $[29,30]$.

Many prodrugs have been developed to improve drug efficacy, absorption, bioavailability, instability, drug delivery, drug specificity, toxicity, membrane transportation, or patient adherence (like poor taste or odor of drug) [31,32]. Designing of prodrugs generally depends upon many factors including the mechanism of action, enzymatic interaction, lipophilicity, functional groups present on the drug and their lability, and metabolic pathway of drug.

There have been major efforts on the development of cytarabine derivatives to generate compounds with higher therapeutic index for the treatment of leukemia and lymphoma (sections 2.1-2.3). Among the explored strategies, the prodrug strategy of introducing modifications on the parent drug to enhance plasma half-life or delivery to cancer cells is a subject of major interest.

The prodrug design for cytarabine has been explored on the basis of mechanism of action and metabolism of the drug. The deamination of the cytosine base of cytarabine by cytidine deaminase leads to formation of uridine derivative (AraU) and deactivation of the drug. In general, cytarabine prodrugs have been designed either to circumvent its catabolism into AraU or to enhance cellular delivery. Some of the prodrug 
approaches included designing amino acid-cytarabine, fatty acid-cytarabine, and amino acid-fatty acid-cytarabine derivatives (sections 2.1 and 2.2).

Mechanistically, cytarabine acts as a prodrug itself and is converted intracellularly to cytarabine triphosphate through monophosphate and diphosphate derivatives before generating biological activities. In the process, monophosphorylation is the rate-limiting step in the metabolic conversion to triphosphate form. Alternatively, many phosphate derivatives of cytarabine have been evaluated as prodrugs to improve the cellular uptake of active metabolites (section 2.3).

\subsection{Amino acid derivatives}

Many amino acid and peptide derivatives are known to increase the cellular uptake of the drug molecules. Peptides with both nucleophilic group $\left(-\mathrm{NH}_{2}\right)$ and electrophilic $(-\mathrm{COOH})$ groups can be used for the conjugation of a large number of drug molecules. Amino acid or peptide attached to a drug can reduce the undesired metabolism by modification of the hydrolytic activity or the steric effect surrounding susceptible functional groups. Cytarabine derivatives substituted with amino acids at 4amino or 5'-hydroxyl positions have been synthesized and evaluated by different groups as described below.

The amino acid-substituted derivatives of cytarabine at 4-amino position were designed to reduce the metabolism of cytarabine into uridine derivative by blocking the proximity of cytidine deaminase. Derivatization of cytarabine with a single amino acid at amino group reported by Jin et al. [33] involved the protection of hydroxyl groups by tert- 
butyldimethyl silyl (TBDMS) followed by the conjugation with protected amino acids. Amino acid derivatives synthesized with arginine, leucine and isoleucine (Figure 2) were evaluated for cellular uptake in Caco-2 cells and were compared with that of cytarabine. The arginine derivative was shown to have comparable uptake while the leucine and isoleucine derivatives reduced the uptake of drug. They have also compared the concentration dependency cellular uptake of the synthesized derivatives and found a similar uptake for cytarabine and arginine-cytarabine at increased concentrations while leucine-cytarabine and isoleucine-cytarabine derivatives showed less cellular uptake than that of the parent drug at higher concentrations.
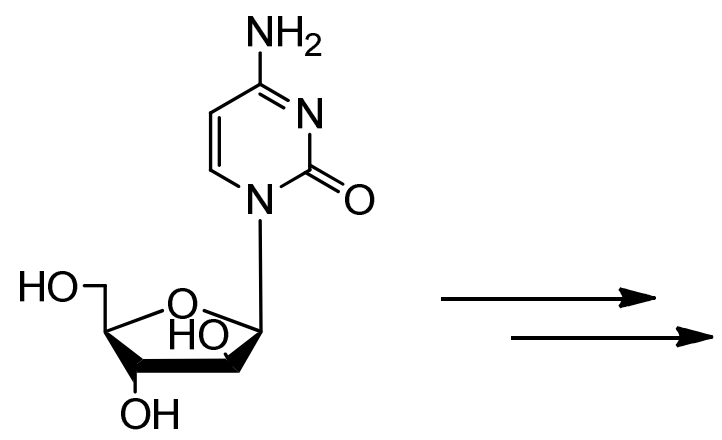

$\mathrm{R}=$ Arginine, Leucine, Isoleucine

Figure 2. N4-substituted amino acid derivatives of cytarabine.

Sun et al. reported the $5^{\prime}$-amino acid derivatives of cytarabine and compared their cellular uptake in Caco-2 and HELA cells [34] (Figure 3). Among the various amino acid derivatives, the 5'-valyl prodrug exhibited highest permeability across the Caco-2 cells monolayer followed by the $5^{\prime}$-isoleucine derivative. Compared to L-Isomer of valine, D-isomer had significantly less cellular uptake, suggesting that the 
stereochemical oligopeptide transporters may be involved in transporting amino acid prodrugs. Antiproliferative studies on the HL-60 cells showed the comparable activity of amino acid derivatives versus that of parent cytarabine. The antiproliferative effects of cytarabine and the amino acid ester prodrugs $\left(\mathrm{IC}_{50}\right)$ in $\mathrm{HL}-60$ Cells were reported as cytarabine $(20 \mu \mathrm{M}), 5^{\prime}$-L-valyl-cytarabine $(16 \mu \mathrm{M}), 5^{\prime}$-D-valyl-cytarabine $(20 \mu \mathrm{M}), 5^{\prime}$-Lisoleucyl-cytarabine $(21 \mu \mathrm{M}), 5^{\prime}$-L-phenylalanyl-cytarabine $(17 \mu \mathrm{M}), 5^{\prime}$-D-phenylalanylcytarabine $(16 \mu \mathrm{M})$, and $5^{\prime}$-L-tryptophyl-cytarabine $(19 \mu \mathrm{M})$. The study recommended the valyl prodrug as the potential candidate for the oral delivery of cytarabine in a similar fashion the other valyl prodrugs that have been reported to be promising with other nucleoside drugs for other conditions [35].
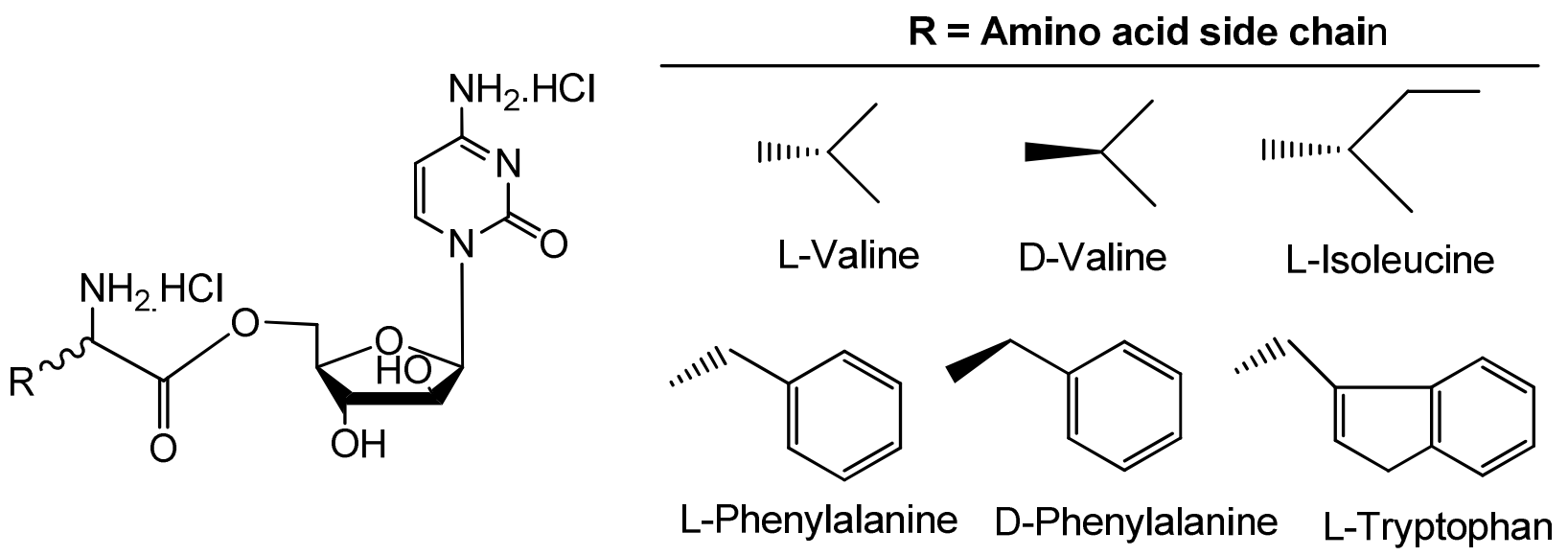

Figure 3. Structure of 5'-amino acid ester prodrugs.

\subsection{Fatty acid derivatives}

The pharmacological properties of drugs depend upon overall surface interactions with medium and can be modulated by variation in the 
lipophilicity/hydrophilicity ratio. The presence of long fatty acyl chains makes the drug molecules lipophilic [36]. Furthermore, the lipophilic drugs pass across the membrane rapidly and make the drug available for biological action at higher intracellular concentration in target cells. The fatty acid conjugation of the drugs through esterification or amidation leads to formation of ester or amide bond, respectively, with the drug molecule. The approach has two advantages: First, the conjugation protects the functional group. Second, the substitution allows sustained and continuous release of the drug through hydrolysis thus minimizing the side effects at higher doses of drugs.

The prodrug approach using fatty acid derivatization at 5'-OH and 4- $\mathrm{NH}_{2}$ groups of cytarabine has been evaluated by many research groups. In addition to making the drug more lipophilic, the strategy may also allow the protection of cytarabine from cytidine deaminase enzyme action and thus preventing its metabolism into inactive form AraU.

Fatty acid presence along with amino acid further utilizes the assumed properties of amino acid as well as fatty chains. Liu. et al. has used the advantage of simultaneous amino acid and fatty acid derivatization [37]. They reported N4-amino acid (Val, Met, Tyr, Glu and Arg) and fatty acid derivatives with chain length of 10,14 and 18 carbons (Figure 4). The synthesis was accomplished through the direct coupling of amino acidfatty acyls derivatives and cytarabine at 4- $\mathrm{NH}_{2}$ (i.e., N4) position through a peptide bond. The synthesized derivatives showed variation in anti-proliferative activities with different amino acids and/or fatty acid. The studies performed using HL-60 and HELA cells showed potential reduction in $\mathrm{IC}_{50}$ with the substitution at N4. Among the different amino acids derivatives, the methionine derivative demonstrated better antiproliferative 
activity when compared to the other synthesized conjugates. The antiproliferative activity was dependant on the chain length and decreased with increase in chain length at N4, suggesting that introduction of bulky groups at N4 through amide bond would not be beneficial in improving biological activity.

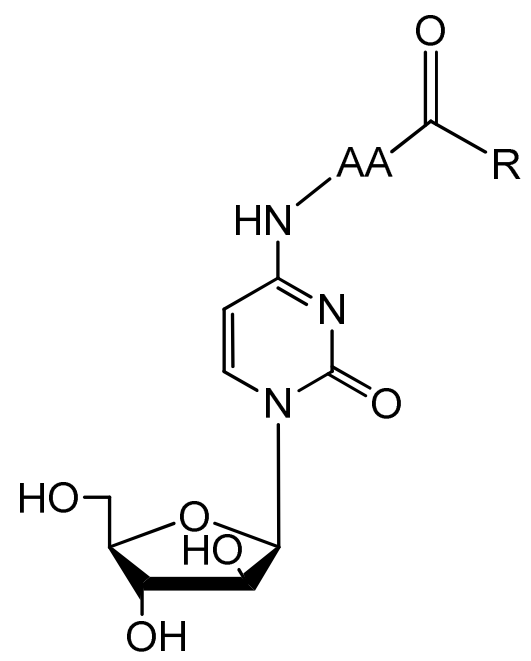

$$
\begin{aligned}
& \mathrm{AA}=\mathrm{Val}, \text { Met, Tyr, Glu, Arg } \\
& \mathrm{R}=-\left(\mathrm{CH}_{2}\right)_{8} \mathrm{CH}_{3},-\left(\mathrm{CH}_{2}\right)_{12} \mathrm{CH}_{3},-\left(\mathrm{CH}_{2}\right)_{16} \mathrm{CH}_{3}
\end{aligned}
$$

Figure 4. Structure of fatty acid-amino acid-cytarabine derivatives.

Fatty acids-substituted conjugates of cytarabine at 5'-OH showed significantly different behavior than N4 derivatives. Derivatization with different saturated and unsaturated higher fatty acids showed comparable activity to that of cytarabine in different cell lines [38]. Among the derivatives, CP-4055, a fatty acid derivative of cytarabine (ara-C-5'-elaidic acid ester) (Figure 5), facilitated and enhanced cellular accumulation and retention of ara-C in tumor cells. Unlike cytarabine, the cellular uptake of CP-4055 is independent of nucleoside transporters, and is believed to be via passive diffusion through the cellular membrane or an alternative internalization mechanism 
[39]. CP-4055 is then hydrolyzed intracellularly by esterases to release free cytarabine, which is subsequently phosphorylated to the active triphosphate analog. When compared with cytarabine as a potent inhibitor of DNA synthesis [40], CP-4055 also transiently inhibits RNA synthesis. Furthermore, the fatty acyl conjugation potentially delayed inactivation to ara-U. CP-4055 is not a substrate for cytidine deaminase. CP4055 demonstrated cytotoxicity in solid tumor and leukemia cells in vitro and in vivo [41]. CP-4055 was evaluated against a lymphoma cell line with deficient nucleoside transport (5CEM-araC/C8) and was resistant to cytarabine. CP-4055 was able to kill a high proportion of drug-resistant cells by apoptosis [42]. Further studies of CP-4055 in human leukemia and lymphoma HL-60 and U937 cells demonstrated additive or synergistic cytotoxic activity in combination with other anticancer drugs, such as cloretazine, idarubicin, gemcitabine, irinotecan, and topotecan, [43].

Continued treatment with CP-4055 exhibited enhanced antitumor effect in human leukemia and in solid tumor models in vivo. The elaidic acid derivative of cytarabine was studied in phase 1 studies in patients with solid tumors, such as malignant melanoma, ovarian cancer, and non-small cell lung cancer. The pharmacokinetic study in 34 patients demonstrated that at dose levels of $>150 \mathrm{mg} / \mathrm{m}^{2}$ after $0.5-2 \mathrm{~h}$ intravenous infusion, CP-4055 was well tolerated and remained in plasma for up to 5-10 $\mathrm{hr}$ [44]. CP4055 under the brand name Elacyt ${ }^{\mathrm{TM}}$ has now moved to phase 3 trials (NCT01147939) by Clavis Pharma in patients with late stage AML. 


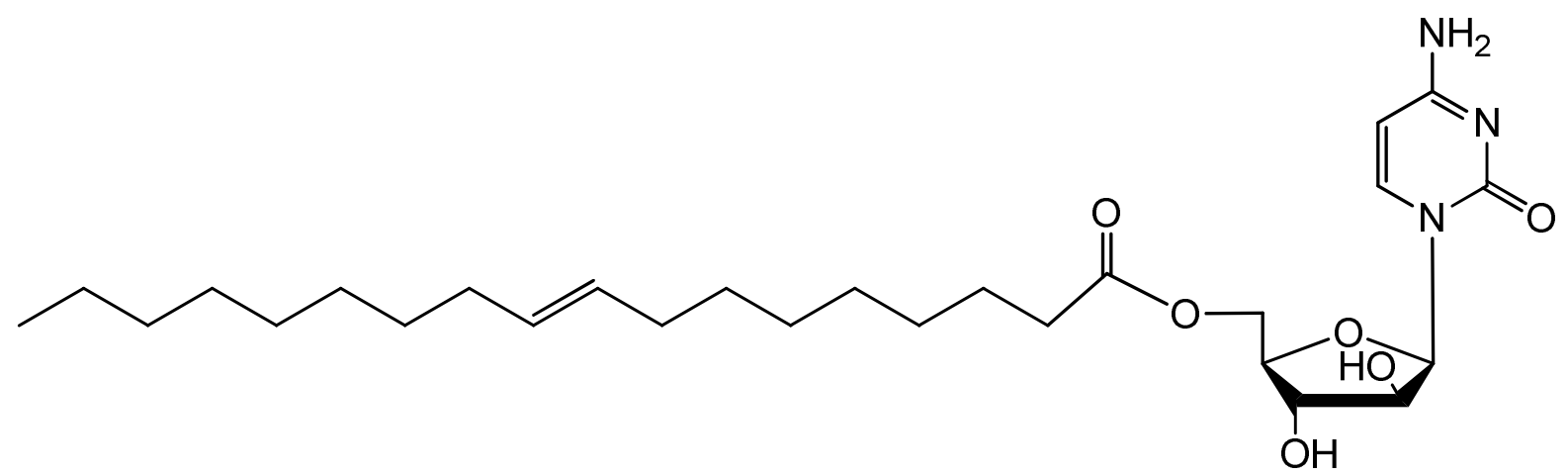

Figure 5. Structure of elaidic acid-cytarabine conjugate (CP-4055).

The 2'-hydroxyl position of arabinose sugar in cytarabine was further explored for functionalizing with fatty acids. We synthesized and evaluated the $2^{\prime}-O$ and $5^{\prime}-O-$ myristoyl and 2',5'-dimyristoyl derivatives (Figure 6). As the cytotoxic mechanism of cytarabine involves phosphorylation of $5^{\prime}$-hydroxyl into triphosphate, the effectiveness of fatty acyl ester derivatives depends on the concentration of the parent drug delivered intracellularly after hydrolysis and availability of $5^{\prime}$-hydroxyl group for phosphorylation [45].
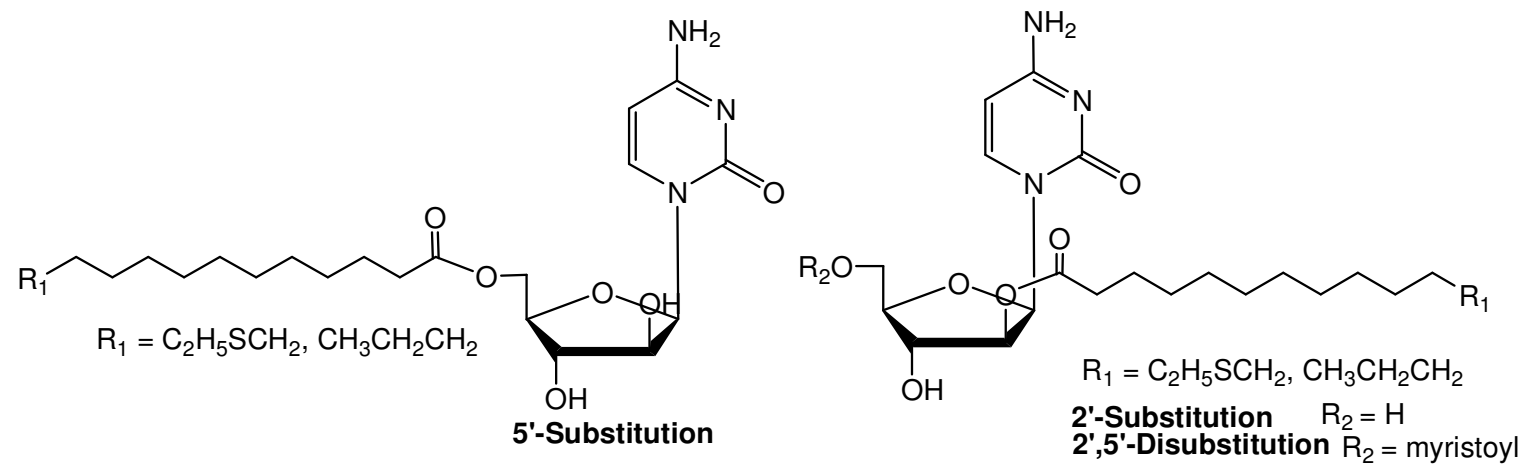

Figure 6. Chemical structure of 5'-O-, 2'-O-monosubstituted and disubstituted cytarabine derivatives. 
The $5^{\prime}$-substituted fatty acyl derivative of cytarabine was not able to inhibit significantly the proliferation of leukemia cells (CCRF-CEM) even after $96 \mathrm{~h}$ at a concentration of $1 \mu \mathrm{M}$. On the other hand, $2^{\prime}, 5^{\prime}$-dimyristoyl derivative of cytarabine and 2 '-fatty acyl derivatives of cytarabine inhibited the growth of cancer cells by approximately by $\sim 36-76 \%$ at a concentration of $1 \mu \mathrm{M}$ after $96 \mathrm{~h}$ incubation. $2^{\prime}, 5^{\prime}-$ Disubstituted derivative showed comparable activity to that of parent drug cytarabine and physical mixtures after $96 \mathrm{~h}$. Enhanced cytotoxic activity of $2^{\prime}, 5^{\prime}$-disubstituted derivative after $96 \mathrm{~h}$ compared to that in $24 \mathrm{~h}$ indicate that the conjugate releases cytarabine slowly and may behave as a prodrug for sustained delivery of the parent nucleoside. Although the anticancer activity of the compound was only comparable to that of cytarabine after $96 \mathrm{~h}$, the sustained-release property may be beneficial.

\subsection{Phosphate derivatives}

The phosphate prodrug approach has been widely used with nucleoside drugs [46]. Nucleosides are converted intracellularly into the monophosphate, diphosphate, and triphosphate derivatives, respectively, before generating biological activities. The monophosphorylation is generally the rate-determining step and thus delivery of monophosphorylated nucleosides that bypass this step is highly desired. Cytarabine is also converted into the triphosphate form before incorporation into nucleic acids. Thus, various phosphate derivatives of cytarabine have been synthesized and evaluated as discussed below.

Lipophilic phosphate prodrugs of cytarabine were designed to contain both the phosphate and lipid (fatty chain) properties. The concept of using lipophilic nucleoside 
monophosphate ester derivatives of cytarabine was proposed to bypass the first phosphorylation step and circumvent tumor resistance associated with decreased deoxycytidine kinase activity via kinase bypass. Furthermore, it was expected that metabolic degradation by cytidine deaminase to be reduced. Finally, partial masking of the phosphate charge and increased lipophilicity may allow higher intracellular levels of the parent analog $[47,48,49]$.

Initially, it was expected that cleavage of the prodrug moiety was due to the action of phosphodiesterase; however, it was subsequently demonstrated that the release of the parent drug occurs via successive two-carbon degradation via oxidation in peroxisomes [50]. The investigations led to discovery of the stearyl phosphate diester of cytarabine (cytarabine ocfosfate, YNK01) (Figure 7) [51]. Further clinical evaluation of cytarabine ocfosfate showed enhanced stability and higher half-life when compared with cytarabine and showed great promise for oral administration [52,53,54]. A phase $1 / 2$ study demonstrated that $15.8 \%$ of the total dose was absorbed and metabolized to cytarabine and AraU [55]. Cytarabine ocfosfate was approved in 1992 [56] in Japan by Nippon Kayaky under license from Yamasa Shoyu for use in patients with adult acute nonlymphocytic leukemia and myelodysplastic syndrome [57].

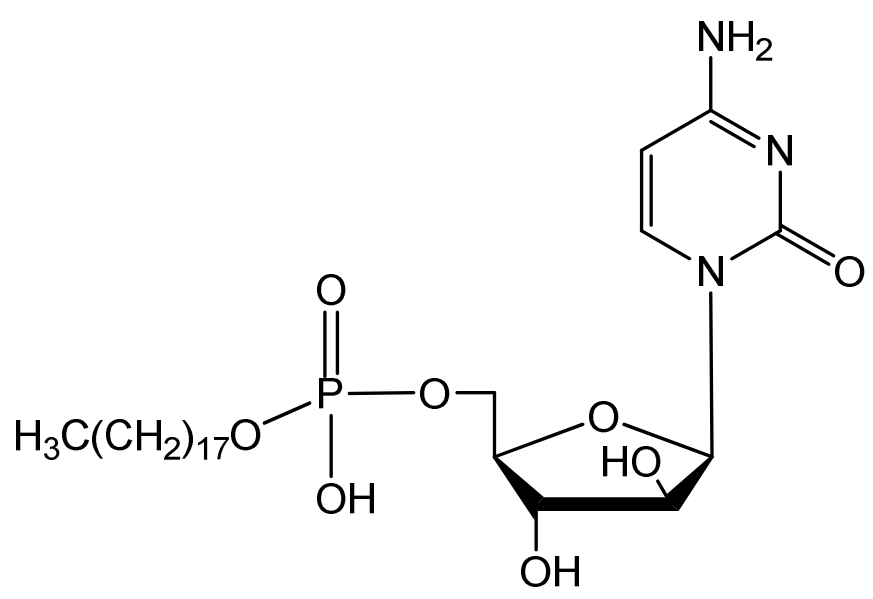


Figure 7: Chemical structure of cytarabine ocfosfate.

Among other phosphate derivatives, the $5^{\prime}$-protected phosphate triester derivatives of cytarabine have been also evaluated as prodrugs. Gouy et al. synthesized mixed phosphate triester derivatives of cytarabine [58] (Figure 8), which were expected to release intracellularly cytarabine monophosphate after esterase- and phosphodiesterase-mediated hydrolysis, respectively (Figure 9).

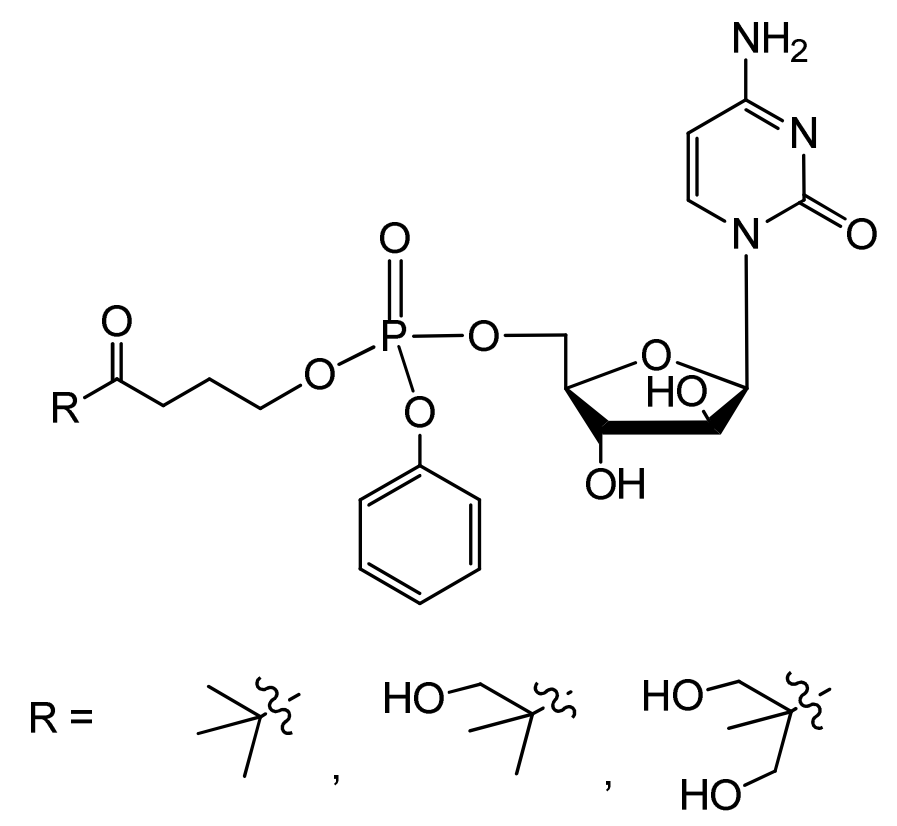

Figure 8. Chemical structures of mixed phosphate araC prodrugs.

Whereas other phosphotriester derivatives with other nucleoside drugs, such as AZT containing S-acyl-2-thioethyl (SATE) phenyl pronucleotides, have shown improvement in activity versus their parent nucleosides, these protected phosphate triester derivatives showed reduced activity against the leukemia cell lines when compared with that of cytarabine. The reduced antitumor activity observed with phosphotriester derivatives of cytarabine was assumed to be a result of the limited 
cellular uptake, a reduced hydrolysis to the nucleoside monophosphate, extracellular hydrolysis to nucleoside monophosphate, or an unexpected decomposition mechanism that hampered the intracellular formation of the $5^{\prime}$-mononucleotide (araCMP).

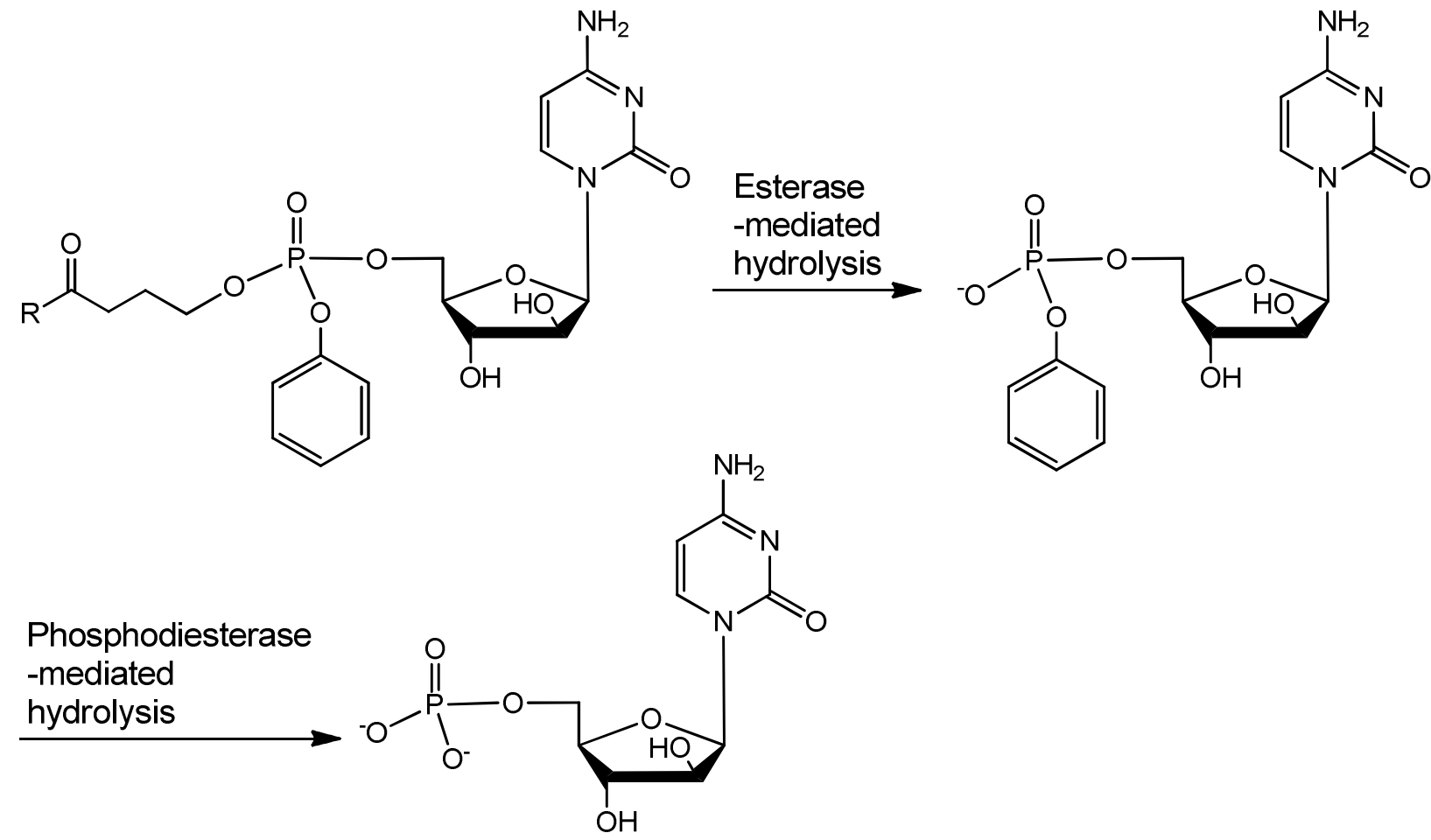

Figure 9. Possible mechanism of hydrolysis of a phosphotriester prodrug of cytarabine into a monophosphate derivative.

To compare the hydrolysis of phosphotriester derivatives of nucleosides [58], three derivatives, cytarabine phosphotriester derivative, AZT phosphate prodrug, and snake venom phosphodiesterase (SVP)-substrate ( $p$-nitrophenyl thymidinyl phosphate), were tested in presence of SVP (snake venom phosphodiesterase), a representative for the type I phosphodiesterase enzyme family at $37^{\circ} \mathrm{C}$. The hydrolysis led to the formation of the corresponding nucleoside 5'-monophosphate (Figure 9) with half-lives of $4.6 \mathrm{~h}$ for cytarabine derivative, $53 \mathrm{~min}$ for AZT derivative, and 43 min for $p$-nitrophenyl thymidinyl phosphate. Thus, 2'-deoxynucleotides were hydrolyzed faster than the corresponding ribonucleotides, and the araC metabolite appeared to be a poor 
substrate for SVP when compared to AZT or thymidine. These data suggest that a slow hydrolysis to cytarabine monophosphate contributes to reduced antitumor activity.

Phosphoramidate derivatives of cytarabine were also shown to have reduced anticancer activity when compared with cytarabine. For example, a nitrofuran phosphoramidate derivative (Figure 10) reported by Tobias et al. showed reduction in anti-proliferative activity when compared to that of cytarabine [59].

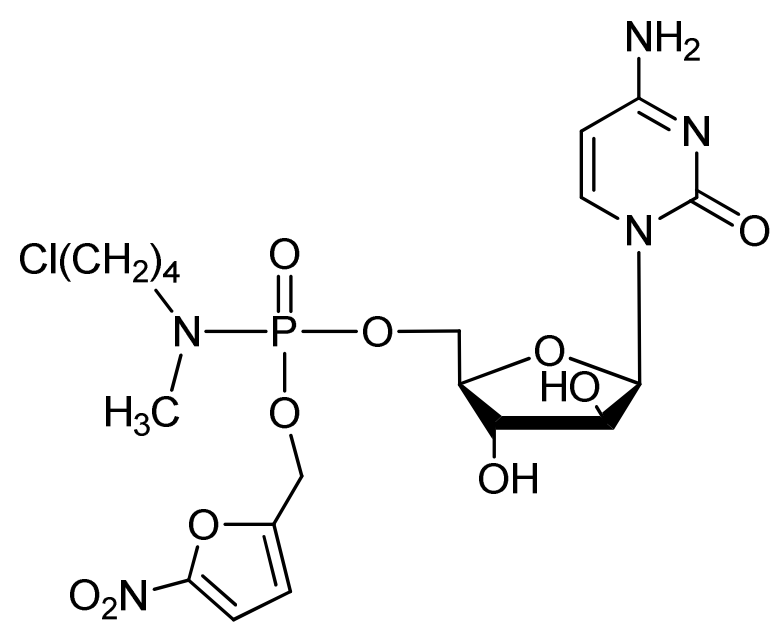

Figure 10. Chemical structure of a cytarabine nitrofuran phosphoramidate.

Phosphoramidate derivatives of cytarabine metabolite $2-\beta-D$-arabinouridine (AraU) (Figure 11) were evaluated by Mehellou et al. [60]. The rationale for this study was to determine whether phosphorylation of AraU can activate the inactivate metabolite. Thus masked phosphate prodrug was synthesized for intracellular delivery. The compounds found to have reduced activity when compared with AraU. Hydrolysis studies in cell extract by NMR showed the reduced activity of phosphoramidate derivatives to hydrolysis because of the stability of P-N bond to hydrolysis. 


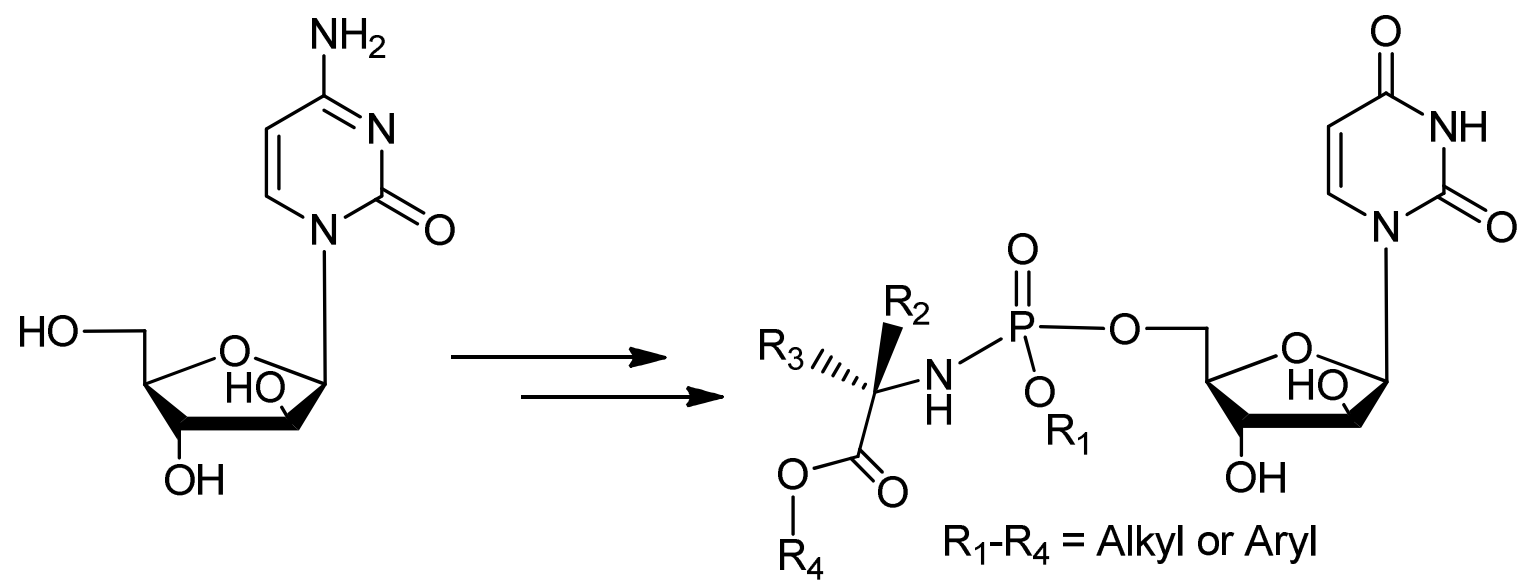

Figure 11. Chemical structure of AraUphosphoramidates.

In general, the biological activities of synthesized fatty acid, amino acid, and phosphate conjugates depended upon the mechanism and ease of intracellularly released cytarabine or monophosphate derivative. The phosphorylation process occurs on the $5^{\prime}-\mathrm{OH}$ group of the arabinose sugar. Prodrug strategies that used either the substitution at 4-amine group of cytosine base or the protected 5'-monophosphate derivatives have resulted in compromised activities against various cancers.

\section{Delivery Systems}

The drugs, which have poor solubility, low cell permeability, fast metabolism and elimination, or require continuous dosages for a longer period of time, could be encapsulated in a protected sheath or fast delivery vehicle without any covalent or chemical modification in drug structure. Various vehicles, sheaths, particles or systems have been introduced as drug delivery systems for improving the delivery of drug molecules to target organ in required dosages. Some of drug delivery systems or 
vehicles include nanoparticles, polymers, hydrogels, organogels, niosomes, and liposomes.

The nucleoside drugs are polar compounds and have generally low membrane permeability. A number of delivery systems evaluated with anticancer nucleoside drugs have been reviewed recently $[61,62]$.

Cytarabine is a highly polar water soluble nucleoside. Various systems have been investigated for encapsulation and targeted delivery of cytarabine. The selection of cytarabine delivery systems [63] depends upon their encapsulating capacity. Some include nanoparticles, hydrogels, chitosan, niosome vesicles of tween 20 , and liposomes. Liposomal cytarabine was approved by the FDA for the treatment of lymphomatous meningitis [64].

\subsection{Hydrogels}

Hydrogels are biomaterials consisting of a polymer matrix with a high water retention capacity. The hydrogels are capable of encapsulating the hydrophilic drugs. Sustained release of the drug depends on the polymer matrix constitution. The hydrogels have application as biomaterials since they have similarity in physical properties, such as water contents, soft consistency and low interfacial tension, to those of natural tissues in biological systems [65]. The encapsulation of hydrophilic drugs such as nucleoside analogs with hydrogels would be easy and loading could be expected higher compared to other polymers. On the other hand, the strong interaction among the polymer and drug would hinder the release. 
Cytarabine has been studied with HEMA (2-hydroxyethyl methacrylate) [66] and HEMA copolymerized with acrylamide [67]. The study showed that the rate and total time of cytarabine release from the copolymer (hydrogels) can be partially modulated by varying the amount of monomer composition and degree of cross linking of the gels. Even though, hydrogels have not been completely successful for delivery of cytarabine, more studies with different polymer compositions can be used in finding the balanced system for drug encapsulation as well as sustained release.

\subsection{Liposomes}

Liposomes or lipid capsids have been important vehicles for the delivery of a number of drugs [68]. The liposomes are lipophilic systems and can pass the cell membranes easily [69]. They possess unique pharmacokinetic characteristics because of their nanometer size (ranges in mean diameter from 50 to $250 \mathrm{~nm}$ ) as systemically administered vesicles [70]. These unique characteristic properties allow clearance via the reticuloendothelial system, which leads to a relatively long systemic circulation time, and hepatic and splenic distribution. Furthermore, the liposomes exhibit preferential extravasation and accumulation at the site of solid tumors due to increased endothelial permeability and reduced lymphatic drainage in these tissues [71]. The increased endothelial permeability has been defined as enhanced permeability and retention effect [72].

Thus, liposomal delivery is a means to modify the pharmacokinetic and pharmacodynamic properties of anticancer agents [73], improving pharmacological properties, and reducing or modulating their toxicity profile [74]. Polar drugs once 
encapsulated inside the liposomes are transported across the membranes by facilitated mechanism of liposome capsid. Liposomal cytarabine formulation has been studied with different liposomal compositions and hybrid systems with polymers [75].

The cytarabine encapsulated liposome has been studied with thermosensitive hydrogels [76]. The hydrogel was based on the biodegradable chitosan and $\beta$ glycerophosphate (C-GP) that are thermosensitive and act as a thermal trigger to regulate the drug release from encapsulation. Combining of liposomes with C-GP hydrogel system gave the advantages of more sustained release of cytarabine along with a higher stability of the hydrogel-liposome system compared to liposome alone. The investigators suggest hydrogel system is appropriate for controlled and sustained release of cytarabine.

Cytarabine crosses the blood-brain barrier only to a limited extent after rapid i.v. administration. Liposomes with encapsulated cytarabine showed enhanced half-life and were found to be suitable for the treatment of lymphomatous meningitis. The formulation has been approved for the treatment of lymphomatous meningitis under the brand name (DepoCyt $\left.{ }^{\circledR}\right)$ [77]. DepoCyt® formulation is prepared by encapsulation of a sterile suspension of cytarabine into multivesicular lipid based polymeric liposomal particles composed of cholesterol, triolein, dioleoylphosphatidylcholine (DOPC) and dipalmitoylphosphatidylglycerol (DPPG). DepoCyt $\AA$ is administered as intrathecal injection in cerebrospinal fluid for the treatment of neoplastic meningitis as a sustainedrelease formulation that maintains cytotoxic concentrations of cytarabine in cerebrospinal fluid for more than 14 days following a single injection $[78,79]$. 
Cytarabine has been used in the combination with anticancer drugs for synergistic antitumor activity. The combination of anticancer drugs as a cocktail is now commonly used clinically in cancer chemotherapy. Alternatively, two or more drugs can be encapsulated in the liposomes to enhance the delivery. The combination of cytarabine with daunorubicin $\mathrm{HCl}$ (a DNA intercalator) in 5:1 ratio in a liposomal formulation (cytarabine/daunorubicin $\mathrm{HCl}$ liposome injection) has been used by intravenous infusion for synergistic therapy in the leukemia treatment as CPX-351 [80]. The liposome membrane is composed of distearoylphosphatidylcholine (DSPC), distearoylphosphatidylglycerol (DSPG), and cholesterol in a 7:2:1 molar ratio. The liposomal formulation (CPX-351) demonstrated enhanced therapeutic activity in preclinical tumor models [81] and promising anticancer activity in a phase I clinical trial in patients with hematological malignancies [82,83].

The substituted derivatives of cytarabine have also been encapsulated in liposomes to improve the delivery and anticancer activity. Schwendener et al. studied the encapsulation of N4-fatty acyl derivatives in liposomes and evaluated their potency compared to cytarabine liposomes [84]. The lipophilic N4-hexadecyl cytarabine (a potent derivative among N4-fatty acyl derivatives) was encapsulated in liposomes and evaluated in a cologenic assay with HL-60 cells showing comparable activity with cytarabine. On the other hand, the liposomal formulation showed higher cytotoxicity in cytarabine resistant cell line compared to that of parent cytarabine [85]. The cellular uptake of liposomal cytarabine derivative was found to be five times more than cytarabine [86]. Similarly among other derivatives of cytarabine N4-octadecyl cytarabine 
also showed a similar behavior. After intravenous injection, the liposomal formulation showed longer plasma half-life compared to cytarabine $[87,88]$.

\subsection{Nanoparticles}

Nanometer size particles have higher delivery through enhanced permeability to the tumor sites [89]. Drugs attached or encapsulated are delivered at higher concentrations to the site of action and are protected from fast excretion [90]. The polymeric nanoparticles have been extensively investigated for the delivery of various drugs $[91,92]$.

Rukmani et al. investigated the methacrylic acid polymeric nanoparticles encapsulation of cytarabine [93]. Detailed morphological analysis of nanoparticles and further in vivo study of hematological parameters after administering CTH nanoparticles continuously for 9 days to tumor bearing mice showed improvement in lifespan of leukemia mice by the formulation when compared to cytarabine alone. The nanoparticular cytarabine formulation was able to restore the altered physiological parameters of leukemic mice to the normal levels and found to have improved pharmacokinetics profile and prolonged the lifespan of mice with increased white blood cells count that make the nanoparticle a potential carrier for the cytarabine.

\subsection{Other Delivery systems}

Among other systems that can serve as delivery vehicles, the natural or artificial polymers/molecules that are biocompatible have been evaluated to improve the delivery of the drug. Some systems include chitosan [94] and niosome vesicles [95] of tween-20 
and tween-80 $[96,97]$. With these systems little success has been made in getting the final applicable system because of insufficient release of the drug from the conjugates.

In conclusion, among the delivery systems evaluated for application of cytarabine, liposomal formulation has been successful in increasing the half-life and found application in treatment of lymphomatous meningitis. The other systems have shown promising improvement in delivery and require more systemic study for final application.

\section{Conclusion}

Cytarabine, a polar nucleoside antileukemia drug with limited plasma stability and cell permeability, has been extensively evaluated by using prodrug and delivery systems. The prodrug attempts to improve delivery to target tissues, plasma stability, and therapeutic effect have been successful in generation of new drug conjugate molecules. Based on these concepts, a number of prodrugs and formulations were designed for cytarabine. Prodrug strategies included conjugation with fatty acids, amino acids, and substituted-phosphate derivatives of cytarabine. The activities of these conjugates were dependent on the rate of hydrolysis, cellular uptake, and resistance to cytidine deaminase. Among the prodrugs amino acid derivative ValCytarabine and fatty acid derivative CP-4055 (under Phase II trials) are being evaluated for the treatment of leukemia and solid tumors, respectively. Among lipophilic phosphodiester derivatives, cytarabine ocfosfate was approved for leukemia treatment. Furthermore, many delivery systems for improving cellular permeability, encapsulation, and the sustained release of cytarabine have been evaluated. Some include hydrogels, liposomes, niosomes, 
nanoparticles, and polymers. The liposomal-cytarabine formulation (DepoCyt®) has been approved for the treatment of lymphomatous meningitis. Both prodrug and delivery systems have been effective in improving the biological profile of cytarabine. These approaches provide insights for designing prodrugs or delivery systems for other anticancer nucleoside drugs. Future investigations are required to generate additional prodrugs and delivery systems with optimal biological activity and delivery. Furthermore, a combination of using an optimal cytarabine prodrug with an appropriate delivery system or using cytarabine or cytarabine prodrugs in conjunction with different anticancer regimens and/or delivery systems may be needed to explore further applications of these strategies in anticancer research.

\section{Expert Opinion}

The concept of prodrug has been successfully used in improving the delivery, stability and pharmacological properties of drugs. The prodrug design is based on direct molecular changes on the drug to generate a dormant compound with appropriate physicochemical properties and desired biological activities. In case of nucleoside analogs, the prodrug designing concept is also used to bypass rate-limiting metabolic process by delivering nucleotides. It is not always easy to predict whether the dormant prodrug will be successful in generating desired properties. As shown with many designs for cytarabine prodrugs, modulating higher stability and better release in target tissues still remain a major challenge. Designing diverse number of prodrugs has allowed the discovery of compounds with optimal stability and release properties. 
Nucleoside analogs have sugar and base moieties. The conjugation at appropriate positions is required for designing optimal prodrugs. Cytarabine has an arabinose sugar with three hydroxyl groups and a base with a free amino group. These functional groups generate high polarity and a limited cellular uptake. Furthermore, some of the functional groups, such as 4-amino and 5'-hydroxyl groups, are critical in stability and activation of the nucleoside, respectively. Cytarabine is inactivated by deamination of the base. Thus, some of the methods used for designing prodrugs of other anticancer or antiviral nucleosides may not be used here since additional care must be taken to prevent deamination. On the other hand, cytarabine is activated by conversion into nucleotides through phosphorylation at 5' position. The designed prodrugs of cytarabine used the attachment of functional moieties at either the amino group of the base or 5'-hydroxy group arabinose sugar. The initial modifications used the fatty acids and different amino acids to improve the delivery and protection from deamination.

The rationale behind 4-N modification with introducing the amide group and bulky fatty acids and amino acids was to block enzyme-mediated deamination into uridine. Although the stability of the compounds were enhanced by reducing deamination, modifications at N4 position led to reduction of activity of the compounds compared to the parent analog. A less unhydrolyzable amide and the presence of a bulky group (amino acids alone and/or with fatty acid) at position 4 made the parent drug less active possibly due to the presence of steric hindrance at the base during incorporation in nucleic acids. Thus, for improving the stability with $\mathrm{N}-4$ substitution may not generate prodrugs with optimal biological properties. 
Alternatively, introduction of functional moieties at $5^{\prime}-\mathrm{OH}$ involved with hydrolyzable ester groups gave products with improved pharmacological properties. The $5^{\prime}$-fatty acid derivative (Elacyt ${ }^{\mathrm{TM}}$ ) is under phase 3 clinical trials for anticancer studies (NCT01147939) by Clavis Pharma.

Furthermore, prodrugs with a substituted phosphate group were expected to enhance biological activity by bypassing the first intracellular rate-limiting phosphorylation step. A simple lipophilic phosphate diester prodrug, cytarabine ocfosfate, was approved in 1992 for leukemia treatment. However, the phosphoramidate derivatives with stable $\mathrm{P}-\mathrm{N}$ bond showed reduced antiproliferative activity in most of the cancer cells including leukemia and solid tumor lines. Thus, less stable phosphoester prodrugs are preferred to amidate counterparts for designing the next generation of lipophilic phosphate prodrugs of cytarabine.

Less research has been carried out on conjugation of cytarabine with hydroxyl groups at $2^{\prime}$ and $3^{\prime}$ positions. It appears that substitution at $5^{\prime}$-position with fatty acids alone is beneficial in generating higher lipophilicity and cellular uptake, but may slow down the metabolic phosphorylation of cytarabine. Alternatively, it may be possible to design prodrugs with masked phosphate at $5^{\prime}$-position and lipophilic chains at $2^{\prime}$ or $3^{\prime}$ positions. It remains to be seen whether this strategy can combine the benefits of both approaches.

Owing to limited cellular uptake of cytarabine, noncovalent methods using delivery systems or vehicles were expected to show great promise. These strategies will protect the chemical integrity of cytarabine and the compound is delivered in an intact 
form. The encapsulation into protected sheaths like polymers, liposomes, hydrogels, and niosomes were expected to provide advantage of membrane transportation and continuous availability in the plasma over time. Among the evaluated delivery systems, the liposomal formulation was successful in enhancing the cytarabine half-life and treatment of lymphomatous meningitis. The hydrogels and niosomes were able to entrap polar cytarabine but the release from these systems remained slow due to strong interaction of drug with the sheath. The future studies need to focus on more optimization of delivery sheaths particularly hydrogel surfaces and nanoparticles to generate controlled or sustained release and appropriate balance of encapsulation and release properties.

It remains to be determined whether an optimal cytarabine prodrug or those under evaluations (e.g., Elacyt ${ }^{\mathrm{TM}}$ ) can be combined with promising delivery systems such as liposomes. Furthermore, cytarabine is also used in combination with other anticancer drugs in treatment of patients. Further studies are required to determine whether combination of cytarabine prodrugs with other anticancer agents or using anticancer cocktails containing cytarabine prodrug or cytarabine with an appropriate delivery system can have any beneficial therapeutic effect in treatment of cancer patients.

In summary, design of cytarabine prodrug derivatives and use of delivery systems have generated prodrugs or formulations that have optimal anticancer activity, stability, or delivery. The exploration of inactive prodrugs or not optimal delivery systems provided deep understanding of the underlying factors responsible for inactivity or incompatibility in formulation that led to the development of first generation of products 
approved by FDA. Further improvement in design, modification in the drug molecule, or delivery sheath may lead to the second generation of compounds with broader applications. The same path may inspire the investigators to design more optimized anticancer nucleosides and other anticancer agents using the knowledge gained here.

\section{Acknowledgement}

We acknowledge the financial support from the American Cancer Society Grant \# RSG-07-290-01-CDD.

\section{Bibliography}

Papers of special note have been highlighted as either of interest $(\cdot)$ or of considerable interest $(\cdot \bullet)$ to readers.

1. Meeker ND, Yang JJ, Schiffman JD. Pharmacogenomics of pediatric acute lymphoblastic leukemia. Expert Opin Pharmacother 2010;11(10):1621-32.

2 Faderl S, Talpaz M, Estrov Z et al. The biology of chronic myeloid leukemia. N Engl J Med 1999;341:164-72.

3. The Leukemia and Lymphoma Society, Facts 2009-2010. Available at http://www.leukemia-lymphoma.org/attachments/National/br_1247234696.pdf [Last accessed July 15, 2010]

4. Fullmer A, O'Brien S, Kantarjian H, Jabbour E. Emerging therapy for the treatment of acute lymphoblastic leukemia. Expert Opin Emerg Drugs 2010;15(1):1-11.

5. Cilloni D, Messa E, Rotolo A, Saglio G. Emerging drugs for chronic myeloid leukemia. Expert Opin Emerg Drugs 2010;15(2):175-84. 
6. Harris TJR, McCormick F. The molecular pathology of cancer. Nat Rev Clin Oncol 2010;7(5):251-65.

7. Kumar L, Vikram P, Kochupilla V. Recent advances in the management of multiple myeloma. Nat Med J Ind 2006;19(2):80-9.

8. Das M, Mohanty C, Sahoo SK. Ligand-based targeted therapy for cancer tissue. Expert Opin Drug Del 2009;6(3):285-304.

9. Fernandez HF, Rowe JM. Induction therapy in acute myeloid leukemia: Intensifying and targeting the approach. Cur Opin Hematol 2010;17(2):79-84.

10. Arya LS. Acute lymphoblastic leukemia: Current treatment concepts. Ind Pediatrics 2000;37(4):397-406.

11. Hoogstraten B, Glidewell O, Holland JF et al. Long term follow-up of combination chemotherapy-radiotherapy of stage III Hodgkin's disease: a Cancer and Acute Leukemia Group B study. Cancer 1979;43(4):1234-44.

12. Kumar R, Shandal V, Shamim SA, Halanaik D, Malhotra A. Clinical applications of $\mathrm{PET}$ and PET/CT in pediatric malignancies. Expert Rev Anticancer Ther 2010;10(5):755-68.

13. Jabbour E, Cortes JE, Giles FJ et al. Current and emerging treatment options in chronic myeloid leukemia. Cancer 2007;109(11):2171-81.

14. Shah M, Agarwal B. Recent advances in management of acute myeloid leukemia (AML). Ind J Pediat 2008;75(8):831-37.

15. Pinilla-lbarz J, Bello C. Modern approaches to treating chronic myelogenous leukemia. Cur Oncol Rep 2008;10(5):365-71.

16. Matsumura I. Standard therapies for acute myeloid leukemia. [Rinshō ketsueki] Japan J Clin Hemat 2009;50(10):1389-1400. 
17. Mayer RJ, Davis RB, Schiffer CA. Intensive postremission chemotherapy in adults with acute myeloid leukemia. N Engl J Med 1994;331:896-903.

18. Robak T, Wierzbowska A. Current and emerging therapies for acute myeloid leukemia. Clin Ther 2009;31(2):2349-70.

19. Abali H, Urün Y, Oksüzoğlu B et al. Comparison of ICE (ifosfamide-carboplatinetoposide) versus DHAP (cytosine arabinoside-cisplatin-dexamethasone) as salvage chemotherapy in patients with relapsed or refractory lymphoma. Cancer Invest 2008;26(4):401-6.

20. Petit J, Boqué C, Cancelas JA et al. Feasibility of ESHAP + G-CSF as peripheral blood hematopoietic progenitor cell mobilisation regimen in resistant and relapsed lymphoma: a single-center study of 22 patients. Leuk Lymphoma 1999;34(1-2):119-27.

21. Weigert $O$, Weidmann $E$, Mueck $R$ et al. A novel regimen combining high dose cytarabine and bortezomib has activity in multiply relapsed and refractory mantle cell lymphoma - long-term results of a multicenter observation study. Leuk Lymphoma 2009;50(5):716-22.

22. Suzuki K, Nakazato T, Sanada $Y$ et al. Successful treatment with hyper-CVAD and highly active anti-retroviral therapy (HAART) for AIDS-related Burkitt lymphoma. Rinsho Ketsueki. 2010;51(3):207-12.

23. Kantarjian HM, O'Brien S, Smith TL et al. Results of treatment with hyper-CVAD, a dose-intensive regimen, in adult acute lymphocytic leukemia. J Clin Oncol 2000;18(3):547-61.

24. Plunkett W, Liliemark JO, Estey E, Keating MJ. Saturation of ara-CTP accumulation during high-dose ara-C therapy: Pharmacologic rationale for intermediate-dose ara-C. Semin Oncol 1987;14:159-66.

25. Cohen SS. The lethality of aranucleotides. Med Biol 1976;54(5):299-326. 
26. Capizzi RL, White JC, Powell BL, Perrino F. Effect of dose on the pharmacokinetic and pharmacodynamic effects of cytarabine. Semin Hemat 1991;28(4):54-69.

27. Slevin ML, Piall EM, Aherne GW, Johnston A, Lister TA. Subcutaneous infusion of cytosine arabinoside. A practical alternative to intravenous infusion. Cancer Chemother Pharmacol 1983;10(2):112-4.

28. Stella VJ, Charman WN, Naringrekar VH. Prodrugs. Do they have advantages in clinical practice? Drugs 1985;29:455-73.

29. Harper NJ. Drug latentiation. Prog Drug Res 1962;4:221-94.

30. Sinkula AA, Yalkowsky SH. Rationale for design of biologically reversible drug derivatives: prodrugs. J Pharm Sci 1975;64:181-210.

31. Peterson LW, McKenna CE. Prodrug approaches to improving the oral absorption of antiviral nucleotide analogues. Expert Opin Drug Del 2009;6(4):405-20.

32. Ali M, Hariharan AG, Mishra N, Jain S. Catalytic antibodies as potential therapeutics. Ind J Biotech 2009;8(3):253-58.

33. Jin MJ, Hong JH, Han HK. Synthesis and in vivo evaluation of N4-amino acid derivatives of Cytarabine for improving the oral delivery of cytarabine. J Kor Pharm Sci 2008;38(4):255-9.

34. Sun $Y$, Sun J, Shi L et al. Synthesis, transport and pharmacokinetics of 5-amino acid ester prodrugs of 1- $\beta$-D-arabinofuranosylcytosine. Mol Pharm 2009;6(1):315-25.

35. Li F, Maag J, Alfredson H. Prodrugs of nucleoside analogues for improved oral absorption and tissue targeting. J Pharm Sci 2008;97(3):1109-34.

36. Nam NH, Sardari S, Selecky M, Parang K. Carboxylic acid and phosphate ester derivatives of fluconazole: Synthesis and antifungal activities. Bioorg Med Chem 2004;12(23):6255-69. 
37. Liu B, Cui C, Duan W et al. Synthesis and evaluation of anti-tumor activities of N4 fatty acyl amino acid derivatives of $1-\beta$-arabinofuranosylcytosine. Eur J Med Chem 2009;44:3596-3600.

38. Bergman AM, Kuiper CM, Voorn DA et al. Antiproliferative activity and mechanism of action of fatty acid derivatives of arabinofuranosylcytosine in leukaemia and solid tumour cell lines. Biochem Pharmacol 2004;67:503-11.

- Study reports 5 '-fatty acyl derivatives of cytarabine and their anticancer activities in different cell lines.

39. Breistøl K, Balzarini J, Sandvold ML et al. Antitumour activity of P-4055 (Elaidic Acid-Cytarabine) compared to cytarabine in metastatic and s.c. human tumour xenograft models. Cancer Res 1999;59:2944-9.

40. Ross DD, Chen SR, Cuddy DP. Effects of 1-beta-D-arabinofuranosylcytosine on DNA replication intermediates monitored by $\mathrm{PH}$-step alkaline elution. Cancer Res 1990;50:2658-66.

41. Bergman AM, Kuiper CM, Myhren F et al. Antiproliferative activity and mechanism of action of fatty acid derivatives of arabinosylcytosine (ara-C) in leukaemia and solid tumour cell lines. Nucleosides Nucleotides Nucleic Acids 2004;23:1523-6.

42. Galmarini CM, Myhren F, Sandvold ML. CP-4055 and CP-4126 are active in ara-C and gemcitabine-resistant lymphoma cell lines. Br J Haematol 2009;144(2):273-5.

43. Adams DJ, Sandvold ML, Myhren F et al. Anti proliferative activity of ELACY (CP4055 ) in combination with cloretazine (VNP40101M), idarubicin, gemcitabine, irinotecan and topotecan in human leukemia and lymphoma cells. Leuk Lymphoma 2008;49(4):786-97.

44. Dueland S, Aamdal S, Lind MJ et al. Intravenous administration of CP-4055 (Elacyt $\left.{ }^{T M}\right)$ in patients with solid tumours. A phase 1 study. Acta Oncologica 2009;48:137-45. 
45. Chhikara BS, Mandal D, Parang K. Synthesis and evaluation of fatty acyl ester derivatives of cytarabine as anti-leukemia agents. Eur J Med Chem 2010 doi:10.1016/j.ejmech.2010.07.024.

46. Peterson LW, McKenna CE. Prodrug approaches to improving the oral absorption of antiviral nucleotide analogues. Expert Opin Drug Deliv 2009;6(4):405-20.

47. Hong $\mathrm{Cl}$, Nechaev A, West CR. Nucleoside conjugates as potential antitumor agents. 2. Synthesis and biological activity of 1-b-D-arabinofuranosylcytosine conjugates of prednisolone and prednisone. J Med Chem 1979;22:1428-32.

48. Raetz CRH, Chu MY, Srivastava SP, Turcotte JG. A phospholipid derivative of cytosine arabinoside and its conversion to phosphatidylinositol by animal tissue. Science 1977;196:303-5.

49. Rosowsky A, Kim SH, Ross J, Wick MM. Lipophilic 5'-alkyl phosphate esters of 1- $\beta$ D-arabinofuranosylcytosine and its N4-acyl and 2,2'-anhydro-3'-O-acyl derivatives as potential prodrugs. J Med Chem 1982;25:171-8.

50. MacCoss M, Ryu EK, Matsushita T. The synthesis, characterization, and preliminary biological evaluation of 1- $\beta$-D-arabinofuranosylcytosine-5'-diphosphate-L-1,2-dipalmitin. Biochem Biophys Res Commun 1978;85:714-23.

51. Yoshida Y, Yamada J, Watanabe T et al. Participation of the peroxisomal $\beta$ oxidation system in the chain shortening of PCA16, a metabolite of the cytosine arabinoside prodrug, YNKO1, in rat liver. Biochem Pharmacol 1990;39:1505-12.

52. Saneyoshi M, Morozumi M, Kodama K et al. Synthetic nucleosides and nucleotides. $\mathrm{XVI}$. Synthesis and biological evaluations of a series of 1- $\beta$-D-arabinofuranosylcytosine 5'-alkyl or arylphosphates. Chem Pharm Bull 1980;28:2915-23.

53. Schleyer E, Braess J, Ramsauer B et al. Pharmacokinetics of Ara-CMP-stearate (YNK01): phase I study of the oral Ara-C derivative. Leukemia 1995;9:1085-90. 
54. Ueda T, Kamiya K, Urasaki Y et al. Clinical pharmacology of 1-beta-Darabinofuranosylcytosine-5'-stearylphosphate, an orally administered long-acting derivative of low-dose 1-beta-D-arabinofuranosylcytosine. Cancer Res 1994;54:109113.

55. Braess J, Freund M, Hanauske A et al. Oral cytarabine ocfosfate in acute myeloid leukemia and non-Hodgkin's lymphoma phase I/II studies and pharmacokinetics. Leukemia 1998;12:1618-26.

56. Heussner P, Willemze R, Ganser A et al. YNK01, an oral cytosine arabinoside derivative in acute myeloid leukemia and chronic myeloid leukemia. Haematol Blood Transfus 1997;38:882-5.

57. Snow B. Drug information: A guide to current resources, second Edition, Scarecrow Press, Inc. Chapter 15, 456-464.

58. Gouy MH, Jordheim LP, Lefebvre I et al. Special features of mixed phosphotriester derivatives of cytarabine. Bioorg Med Chem 2009;17:6340-47.

- This article reports study of comparative enzymatic hydrolysis of the phosphate prodrugs of cytarabine.

59. Tobias SC, Borch RF. Synthesis and Biological Evaluation of a Cytarabine Phosphoramidate Prodrug. Mol Pharma 2004;1(2):112-6.

60 Mehellou Y, Valente R, Mottram H et al. Phophoramidates of 2'- $\beta$-D-arabinouridine (AraU) as phosphate prodrugs; design, synthesis, in vitro activity and metabolism. Bioorg Med Chem 2010;18:2439-46.

61. Utreja P, Jain S, Tiwary AK. Novel drug delivery systems for sustained and targeted delivery of anti-cancer drugs: Current status and future prospects. Curr Drug Del 2010;7(2):152-161.

- Contains details about the anti-cancer drug delivery mainly liposomes and nanoparticular systems. 
62. Diab R, Degobert G, Hamoudeh M et al. Nucleoside analogue delivery systems in cancer therapy. Expert Opin Drug Deliv 2007;4(5):513-31.

- This review discusses utility of different delivery systems useful for the nucleoside drugs in respective therapy.

63. Dubey SK, Pandey A, Mishra R et al. Site directed drug delivery by non-viral mode. Ind J Biotech 2007;6(2):159-74.

64. Kripp M, Hofheinz RD. Treatment of lymphomatous and leukemic meningitis with liposomal encapsulated cytarabine. Int J Nanomedicine 2008;3(4):397-401.

65. Manjanna KM, Kumar TMP, Shivakumar B. Natural polysaccharide hydrogels as novel excipients for modified drug delivery systems: A review. Int $\mathrm{J}$ ChemTech Res 2010;2(1):509-25.

66. Trigo RM, Blanco MD, Teijon JM, Sastre R. Anti-Cancer drug, Ara-C, release from pHEMA hydrogels. Biomater 1994;15(14):1181-6.

67. Sastre RL, Blanco MD, Go'mez C et al. Cytarabine trapping in poly(2-hydroxyethyl methacrylate-co-acrylamide) hydrogels: drug delivery studies. Polym Int 1999;48:84350 .

68. Kaasgaard T, Andresen TL. Liposomal cancer therapy: Exploiting tumor characteristics. Expert Opin Drug Del 2010;7(2):225-43.

69. Alam MI, Beg S, Samad A et al. Strategy for effective brain drug delivery. Eur J Pharma Sc 2010;40(5):385-403.

70. Bhattacharya S, Bajaj A. Advances in gene delivery through molecular design of cationic lipids. Chem Commun 2009;31:4632-56.

71. Rao NM. Cationic lipid-mediated nucleic acid delivery: beyond being cationic. Chem Phys Lipids 2010;163(3):245-52. 
72. Maeda $\mathrm{H}, \mathrm{Wu}$ J, Sawa $\mathrm{T}$ et al. Tumour vascular permeability and the EPR effect in macromolecular therapeutics: a review. J Control Release 2000;65:271-84.

73. Abu Lila AS, Ishida, T, Kiwada H. Targeting anticancer drugs to tumor vasculature using cationic liposomes. Pharma Res 2010;27(7):1171-83.

74. Dhamecha DL, Rathi AA, Saifee M et al. Drug vehicle based approaches of penetration enhancement. Int J Pharma Pharmaceut Sc 2009;1:24-46.

75. Benesch M, Urban C. Liposomal cytarabine for leukemic and lymphomatous meningitis: recent developments. Expert Opin Pharma 2008;9(2):301-9.

- This review has a detailed analysis of reports related to liposomal formulation of cytarabine.

76. Mulik R, Kulkarni V, Murthy RSR. Chitosan-Based Thermosensitive Hydrogel Containing Liposomes for Sustained Delivery of Cytarabine. Drug Devel Ind Pharm 2009;35(1):49-56.

77. Benesch M, Urban C. Liposomal cytarabine for leukemic and lymphomatous meningitis: Recent developments. Expert Opin Pharmacother 2008;9(2):301-9.

78. Phuphanich S, Maria B, Braeckman R, Chamberlain M. A pharmacokinetic study of intra-CSF administered encapsulated cytarabine (DepoCyt@) for the treatment of neoplastic meningitis in patients with leukemia, lymphoma, or solid tumors as part of a phase III study. J NeuroOncol 2007; 81(2):201-8.

79. Jaeckle KA, Batchelor T, O'Day SJ. An Open Label Trial of Sustained-release Cytarabine (DepoCyt ${ }^{\mathrm{TM}}$ ) for the Intrathecal Treatment of Solid Tumor Neoplastic Meningitis. J Neuro-Oncol 2002;57(3):231-39.

80. Bayne WF, Mayer LD, Swenson CE. Pharmacokinetics of CPX-351 (cytarabine/daunorubicin $\mathrm{HCl}$ ) liposome injection in the mouse. J Pharmaceut Sci 2009;98(7):2540-48. 
81. Tardi $\mathrm{P}$, Johnstone $\mathrm{S}$, Harasym $\mathrm{N}$ et al. In vivo maintenance of synergistic Cytarabine:Daunorubicin ratios greatly enhances therapeutic efficacy. Leuk Res 2009;33:129-139.

82. Feldman E, Lancet J, Kolitz JE et al. Phase I study of a liposomal carrier (CPX-351) containing an optimized, synergistic, fixed molar ratio of cytarabine and daunorubicin in advanced leukemias and myelodysplastic syndromes. Am Soc Hematol Ann Meet Blood 2007;110: 909.

83. Lima WS, Tardia PG, Santos ND. Leukemia-selective uptake and cytotoxicity of CPX-351, a synergistic fixed-ratio cytarabine:daunorubicin formulation, in bone marrow xenografts. Leuk Res 2010;34(9):1214-23.

84. Schwendener R, Schott $H$. Lipophilic arabinofuranosyl cytosine derivatives in liposomes. Methods Enzymol 2005;391:58-70.

85 Horber DH, Von Ballmoos P, Schott H, Schwendener RA. Cell cycle-dependent cytotoxicity and induction of apoptosis by liposomal N4-hexadecyl-1- $\beta$-Darabinofuranosylcytosine. British J Cancer 1995;72(5):1067-73.

86 Horber DH, Schott H, Schwendener RA. Cellular pharmacology of a liposomal preparation of N4-hexadecyl-1- $\beta$-D-arabinofuranosylcytosine, a lipophilic derivative of 1$\beta$-D-arabinofuranosylcytosine. British J Cancer 1995;71(5):957-62.

87. Rentsch KM, Schwendener RA, Schott H, Hänseler E. Pharmacokinetics of N4octadecyl-1- $\beta$-D-arabinofuranosylcytosine in plasma and whole blood after intravenous and oral administration to mice. J Pharmacy Pharmacol 1997;49(11):1076-81.

88. Koller-Lucae SKM, Schott H, Schwendener RA. Interactions with human blood in vitro and pharmacokinetic properties in mice of liposomal N4-octadecyl-1- $\beta$-Darabinofuranosylcytosine, a new anticancer drug. J Pharmacol Exp Therap 1997;282(3):1572-80.

89. Jancar J, Douglas JF, Starr FW et al. Current issues in research on structureproperty relationships in polymer nanocomposites. Polymer 2010;51(15):3321-43. 
90. Sandhiya S, Dkhar SA, Surendiran A. Emerging trends of nanomedicine - an overview. Fundamental Clin Pharmac 2009;23(3):263-9.

91. Kumari A, Yadav SK, Yadav SC. Biodegradable polymeric nanoparticles based drug delivery systems. Colloids and Surfaces B: Biointerfaces 2010;75(1):1-18.

92. Surendiran A, Sandhiya S, Pradhan SC, Adithan C. Novel applications of nanotechnology in medicine. Ind J Med Res 2009;130(6):689-701.

93. Rukmani K, Sivakumar M, Kumar SS. Nanoparticular drug delivery system of cytarabine hydrochloride $(\mathrm{CTH})$ for improved treatment of Lymphoma. J Biomed Nanotech 2007;3(1):90-96.

- This study has detailed evaluation of nanoparticle-cytarabine along with in vivo application.

94. Blanco MD, Gomez C, Olmo R et al. Chitosan microspheres in PLG films as devices for cytarabine release. Intern J Pharma 2000;202:29-39.

95. Azeem A, Anwer MK, Talegaonkar S. Niosomes in sustained and targeted drug delivery: some recent advances. J Drug Targeting 2009;17(9):671-89.

- This review provides recent important developments in application of niosomes in drug delivery.

96. Sagar GH, Arunagirinathan MA, Bellare JR. Self assembled surfactant nanostructures important in drug delivery: a review. Ind J Exp Biol 2007;45:133-159.

97. Ruckmani K, Ghosal SK. The efficacy of 1- $\beta$-D-arabinofuranosylcytosine entrapped in niosomes against leukaemia in mice. STP Pharma Sc 2001;11(4):301-3. 


\section{Article Highlights Box}

- 1. Introduction: A brief introduction about leukemia, cytarabine and its applications, mechanism of action and metabolism

- 2. Cytarabine prodrugs: Various prodrug strategies used with cytarabine

- 2.1 Amino acid derivatives: Different amino acid derivatives of cytarabine substituted at 5'-OH and 4- $\mathrm{NH}_{2}$ positions

2.2 Fatty acid derivatives: Fatty acid derivatization of cytarabine at 5'$\mathrm{OH}, 5^{\prime}-\mathrm{OH}$, and 4- $\mathrm{NH}_{2}$ positions

- 2.3 Phosphate derivatives: Various phosphate derivative of cytarabine, such as cytarabine ocfosfate, phosphotriesters, and phosphoramidates

- 3. Delivery systems: The application of various delivery systems for delivery of cytarabine

- 3.1 Hydrogel: Applicability of hydrogels for delivery of cytarabine

- 3.2 Liposomes: Cytarabine liposomes and their application in cancer

- 3.3 Nanoparticles: The nanoparticular system used with a possible application of delivery of cytarabine

- 3.4 Other delivery systems: A brief account of the other delivery systems evaluated with cytarabine

- 4. Conclusion: Concluding remarks

- 5. Expert opinion: General evaluation of strategies used and proposed alternative approaches, future directions 